\title{
Inhibition of FASN expression enhances radiosensitivity in human non-small cell lung cancer
}

\author{
NING ZHAN ${ }^{1}$, BIN LI $^{2}$, XIANGYING XU ${ }^{3}$, JIANYU XU ${ }^{1}$ and SONGLIU HU ${ }^{1}$ \\ ${ }^{1}$ Department of Radiation Oncology, The Third Affiliated Hospital of Harbin Medical University, Harbin, \\ Heilongjiang 150081; ${ }^{2}$ Department of Plastic Surgery, Nanfang Hospital of Southern Medical University, Guangzhou, \\ Guangdong 510515; ${ }^{3}$ The Center Laboratory of Radiobiology, Institute of Cancer Prevention \\ and Treatment of Heilongjiang Province, Harbin, Heilongjiang 150081, P.R. China
}

Received November 11, 2016; Accepted November 21, 2017

DOI: $10.3892 / \mathrm{ol} .2018 .7896$

\begin{abstract}
Fatty acid synthase (FASN) is the key enzyme required for the de novo synthesis of long-chain fatty acids. FASN has been observed to be overexpressed in the majority of cancer tissues, and its expression is associated with a poor prognosis, potentially mediated by resistance to drug or radiation. The present study investigated whether the downregulation of FASN in non-small cell lung cancer (NSCLC) may increase radiosensitivity. A lentiviral vector containing short hairpin RNA targeted to FASN (pSIH-H1-Puro-shFASN) was successfully constructed and transfected into A549 cells to knockdown the gene by RNA interference. pSIH-H1-Puro-shFASN was used as the experimental group, while pSIH-H1-Puro-shGFP was used as a control group. The mRNA expression levels of FASN were determined using quantitative polymerase chain reaction. In addition, cell proliferation was measured using cell counting kit-8 assay, and colony formation assay was performed to determine the radiosensitizing effect of FASN knockdown. The cell cycle distribution and apoptotic rates were analyzed using flow cytometry, while western blot analysis was used to assess the expression of DNA-dependent protein kinase catalytic subunit protein, which is associated with DNA double-strand break (DSB) repair. The results of the present study revealed that NSCLC cells are more sensitive to radiation following the knockdown of FASN. Furthermore, the increased radiosensitivity may be associated with increased proliferation, promotion of apoptosis and cell cycle arrest in the G2/M phase. Furthermore, downregulated FASN expression reduced the levels of DNA DSB repair-associated proteins following treatment with radiation. These results indicate that
\end{abstract}

Correspondence to: Professor Xiangying $\mathrm{Xu}$, The Center Laboratory of Radiobiology, Institute of Cancer Prevention and Treatment of Heilongjiang Province, 150 Haping Road, Harbin, Heilongjiang 150081, P.R. China

E-mail: xuxxyy@sohu.com

Key words: fatty acid synthase, lentivirus-mediated short hairpin RNA, non-small cell lung cancer, radiosensitivity silencing FASN may sensitize NSCLC cells to radiation treatment. Therefore, FASN may be a potential novel therapeutic target to improve the response of NSCLCs to radiation therapy.

\section{Introduction}

In previous years, lung cancer has been a leading cause of mortality globally (1). According to reports from the International Agency for Research on Cancer, the morbidity and mortality rates of lung cancer have been increasing markedly, and lung cancer has become a significant threat to human health (2). Non-small cell lung cancer (NSCLC) accounts for $\sim 85 \%$ of all lung cancer cases globally (3), with the majority diagnosed at intermediate or advanced stages (3). Morbidity is higher among men than women (4). Despite improved laboratory diagnosis, surgery, chemotherapy and radiotherapy techniques, the 5-year survival rate and prognosis of patients with NSCLC remain unfavorable (5). The main cause of treatment failure is local recurrence (4).

Radiotherapy is an important local and regional therapeutic technique. The role of ionizing radiation in radiotherapy is the induction of DNA double-strand breaks (DSBs) and the inhibition of DNA repair (6). Thorough investigation of molecular variations that affect responses to radiation is required to improve the understanding of the differences in radiosensitivity between individuals. Therefore, understanding NSCLC at the molecular level has attracted extensive research efforts. Furthermore, identifying a specific target to enhance radiotherapeutic efficacy and reduce its adverse effects in NSCLC is of notable interest.

The uncontrolled proliferation of a tumor results in the consumption of large amounts of nutritional substances (7). Under pathological conditions, aerobic oxidation and anaerobic metabolism cannot meet the unlimited energetic requirements of tumor cells (8). Therefore, increasing the function of fat metabolism is an important mechanism by which to maintain the energy supply of tumor cells, in addition to increasing glucose availability (9). Dysregulated lipid metabolism is strongly associated with the development, maintenance and metastatic progression of tumors $(10,11)$. Based on this, it is hypothesized that the reduction of fatty acid synthesis may inhibit tumor cell growth and therefore that fatty acid 
synthase (FASN) may be a potential target in the development of anticancer treatments.

FASN is the pivotal enzyme required for the de novo synthesis of fatty acids and represents one of the most commonly overexpressed lipogenic enzymes (12), which are critical to fatty acid synthesis and metabolism. This is thought to be essential for the progression, metastasis, and drug and radiation resistance of tumors (12). In recent years, accumulating evidence has demonstrated that the overexpression FASN is strongly associated with an unfavorable prognosis and treatment resistance in a variety of human neoplasms, including breast (13), bladder (14), nasopharyngeal carcinoma (15), esophageal (16) and pancreatic cancer (17). Although the roles of FASN in tumor progression and treatment resistance have been investigated, there have been no studies of FASN expression in NSCLC and its association with sensitivity to ionizing irradiation.

Although the expression of FASN is upregulated in various types of cancer, its association with radiosensitivity in NSCLC is not well understood. Therefore, the aim of the present study was to construct an effective short hairpin RNA (shRNA) to knockdown FASN in an NSCLC cell line (A549), and to measure the effect of this on cell proliferation, the cell cycle and apoptosis, in addition to assessing radiosensitivity by the analysis of clone formation and DNA repair proteins.

\section{Materials and methods}

Cell culture. The human NSCLC cell line A549 was obtained from the Institute of Cancer Prevention and Treatment, Harbin Medical University. The cells were routinely cultured in RPMI-1640 medium (Invitrogen; Thermo Fisher Scientific, Inc., Waltham, MA, USA) supplemented with $10 \%$ fetal bovine serum (Hyclone; GE Healthcare Life Sciences, Shanghai, China) at $37^{\circ} \mathrm{C}$ in $5 \% \mathrm{CO}_{2}$ incubator.

Lentiviral vectors construction for FASN small hairpin RNA and transfection. To construct a lentiviral vector containing small hairpin RNA targeted to FASN, the FASN fragment was amplified from human genomic DNA (A549 cell line) using PCR (SanTaq Plus PCR kit; cat. no. SK2491; Sangon Biotech Co., Ltd., Shanghai, China) (30 cycles of denaturing at $94^{\circ} \mathrm{C}$ for $30 \mathrm{sec}$, annealing at $60^{\circ} \mathrm{C}$ for $30 \mathrm{sec}$, and elongation at $72^{\circ} \mathrm{C}$ for $30 \mathrm{sec}$ ). Briefly, $1 \mu \mathrm{g}$ of each plasmid DNA and $20 \mu \mathrm{l}$ of Lipofectamine ${ }^{\circledR} 2000$ (Invitrogen; Thermo Fisher Scientific, Inc.) were mixed separately with Optim-MEM medium (Invitrogen; Thermo Fisher Scientific, Inc.) and incubated for 5 min at room temperature. The FASN fragment was then inserted into AgeI and Ecor I enzyme sites of pSIH-H1-Puro vector and sequenced. Two pairs of effective RNA interference sequences, shRNA\#3072 and shRNA\#7377 to target the FASN gene (Thermo Fisher Scientific, Inc.) were designed (DNAMAN, version 6.0) and synthesized (version A30142; Thermo Fisher Scientific, Inc.). The sequence of shRNA\#3072 was 5'-CCCAGGCTGAAGTTTACAA-3' and the sequence of shRNA\#7377 was 5'-GGTCCTTCTACTACAAGCT-3'. At the same time, a corresponding vector pSIH-H1-Puro-shGFP was constructed as the negative control. Then, lentiviral vector DNAs and packaging vectors were transfected into A549 cells. Supernatants containing lentiviruses were harvested $72 \mathrm{~h}$ after transfection, following puromycin (Invitrogen; Thermo Fisher
Scientific, Inc.) selection to obtain positive clones. Infection efficiency was determined using reverse transcription-quantitative polymerase chain reaction (RT-qPCR) assay and western blot analysis.

$R T$ - $q P C R$. Total RNA was extracted from the A549 cells using the E.Z.N.A DNA/RNA isolation kit (Omega Bio-tek Inc., Norcross, GA, USA). Total RNA kit I (Omega Bio-Tek, Inc., Norcross, GA, USA) and cDNA synthesis was performed using the Transcriptor First Strand cDNA Synthesis kit (Roche Diagnostics, Basel, Switzerland), according to the manufacturer's protocols. Amplification conditions consisted of pre-denaturation at $95^{\circ} \mathrm{C}$ for $10 \mathrm{~min}$ followed by 40 cycles of denaturation at $95^{\circ} \mathrm{C}$ for $15 \mathrm{sec}$, annealing at $55^{\circ} \mathrm{C}$ for $60 \mathrm{sec}$ and elongation at $72^{\circ} \mathrm{C}$ for $45 \mathrm{sec}$. qPCR was conducted using the FastStart Universal SYBR-Green Master (Roche Diagnostics) on a thermocycler (Applied Biosystems 7500 Fast Real-Time PCR System; Thermo Fisher Scientific, Inc.). DNA primers were synthesized by Invitrogen (Thermo Fisher Scientific, Inc.) and the sequences were as follows: FASN forward, 5'-GGACCTGACCTGCCGTCTAG-3' and reverse, 5'-GAGGAGTGGGTGTCGCTGTT-3'; GAPDH forward, 5'-TGGTCTCCTCTGACTTCAAC-3' and reverse, 5'-GTG AGGGTCTCTCTCTTCCT-3'. Human GAPDH was used as an internal control. The $2^{-\Delta \Delta \mathrm{Cq}}$ method was used to calculate the relative mRNA expression of FASN (18).

Irradiation. Stably transfected cells and a negative control group were irradiated at room temperature at a dose rate of $2.0 \mathrm{~Gy} / \mathrm{min}$ ( $2 \mathrm{~Gy}$ for $1 \mathrm{~min}$, then $4 \mathrm{~Gy}$ for $2 \mathrm{~min}$ ) of 6-MV X-ray using an ELEKTA Synergy accelerators (Uppsala, Sweden).

Cell cycle analysis. Cell cycle arrest in the presence or absence of irradiation was measured. $1-3 \times 10^{6}$ cells were seeded onto 6 -well culture plates overnight and were attached to the surface of the plate. Following exposure to $6 \mathrm{~Gy}$ X-ray radiation, the cells were harvested by trypsinization, washed twice with phosphate-buffered saline (PBS) and fixed in $70 \%$ cold ethanol overnight at $-20^{\circ} \mathrm{C}$. The cells were then washed twice with PBS. Subsequently, the cells were resuspended in $0.5 \mathrm{ml}$ propidium iodide (PI; cat. no. 340242; BD Biosciences, San Jose, CA, USA) for staining and incubated in the dark for $30 \mathrm{~min}$ at $37^{\circ} \mathrm{C}$. The cell cycle distribution was analyzed using a Coulter EPICS XL flow cytometer (BD FACS Canto II; Beckman Coulter, Inc., CA, USA) and MODFIT software (version: 3.2; BD Biosciences).

Apoptosis assay. To quantify the population of apoptotic cells, an Apoptosis Detection Annexin kit (Vazyme, Nanjing, China) was used. The cells $\left(1-5 \times 10^{5}\right)$ were seeded onto 6 -well plates, with or without subsequent treatment with $6 \mathrm{~Gy}$ X-ray. The culture medium was collected, and the cells were harvested with trypsin and centrifuged for $10 \mathrm{~min}$ at 1,200 x g at room temperature. The cells were then resuspended in $100 \mu \mathrm{l}$ binding buffer and incubated with $5 \mu \mathrm{l}$ Annexin V and $5 \mu \mathrm{l}$ PI for $10 \mathrm{~min}$ at room temperature, followed by the addition of $400 \mu \mathrm{l}$ binding buffer. Finally, the cell sample was analyzed using a Coulter EPICS XL flow cytometer (BD FACS Canto II; Beckman Coulter, Inc., CA, USA) with Diva software (BD Biosciences; version 1.1.3) according to the manufacturer's protocols. 
A

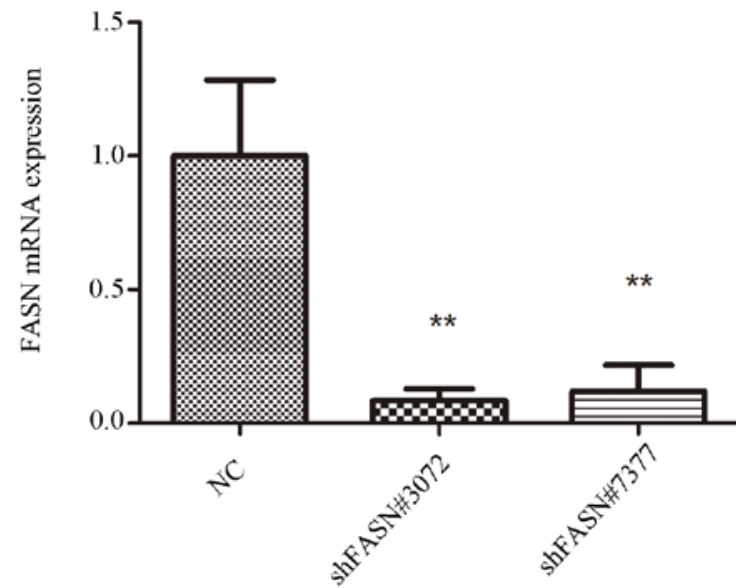

B

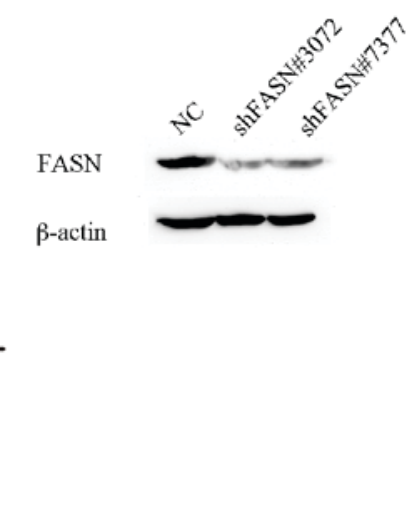

Figure 1. Alteration of FASN expression in the non-small cell lung cancer cell line A549 by lentivirus-mediated RNA interference. A549 cells were stably transfected with vectors containing shFASN\#3072, shFASN\#7377 or NC. (A) Reverse transcription-quantitative polymerase chain reaction analysis of FASN mRNA expression revealed that transfection with shRNA targeting FASN was able to significantly inhibit FASN expression compared with the NC group. ${ }^{* *} \mathrm{P}<0.01$ vs. NC. (B) Western blot analysis of FASN protein expression revealed marked reductions in FASN protein expression levels in the cells that were transfected with shRNA targeting FASN compared with the NC group. FASN, fatty acid synthase; NC, negative control; shRNA, short hairpin RNA.

Colony formation assay. Stable transfected cells were planted on a 6 -well culture plates at different cell densities $(100,200$, $400,1,000$ and 5,000 cells per well). After $24 \mathrm{~h}$, following exposure to a single dose of $0,2,4$ and 6 Gy X-ray radiation with a $100 \mathrm{~cm}$ focus-surface distance at a dose rate of $2.0 \mathrm{~Gy} / \mathrm{min}$ ( $2 \mathrm{~Gy}$ for $1 \mathrm{~min}$, then $4 \mathrm{~Gy}$ for $2 \mathrm{~min}$ ) at room temperature. After treatment, the cells were allowed to grow for 10-14 days to form colonies in an incubator at $37^{\circ} \mathrm{C}$ and $5 \% \mathrm{CO}_{2}$, and then washed with PBS and fixed using methanol for $15 \mathrm{~min}$, and then stained using crystal violet for 10-30 min at room temperature. Subsequently, colonies were counted ( $\geq 50$ cells were scored as clonogenic survivors) under a light microscope (magnification, $\mathrm{x} 40$ ). In each irradiation dose group, surviving fraction of cells was calculated as the plating efficiency of the irradiation cells divided by that of the non-irradiated control. All experiments were performed independently at least three times.

Cell proliferation analysis using cell counting kit-8 (CCK-8). Stably transfected A549 cells were plated onto 96-well plates at a density of $2 \times 10^{3}$ cells $/ 100 \mu \mathrm{l} /$ well. At 1,2 and 3 days after incubation, $10 \mu \mathrm{l}$ CCK-8 solution (Beijing Zoman Biotechnology Co., Ltd, Beijing, China) was added into each well, and the plate was incubated for an additional $2 \mathrm{~h}$ in the dark at $37^{\circ} \mathrm{C}$ in a humidified incubator. The absorbance values for all wells were measured at $480 \mathrm{~nm}$ with a microplate reader (ELx800 Absorbance Reader, BioTek Instruments, Inc., Winooski, VT, USA).

Western blot analysis. The cells were washed twice with PBS and lysed in ice-cold radioimmunoprecipitation assay buffer containing phenylmethanesulfonyl fluoride (Cell Signaling Technology, Inc., Danvers, MA, USA) for $30 \mathrm{~min}$ and centrifuged at $12,000 \mathrm{x}$ g for $15 \mathrm{~min}$ at $4^{\circ} \mathrm{C}$ for isolation of total protein. The protein concentration was determined using a bicinchoninic acid assay (Thermo Fisher Scientific, Inc.). The proteins were then denatured by heating at $99^{\circ} \mathrm{C}$ for $5 \mathrm{~min}$. A total of 20-80 $\mu$ g amounts of protein were loaded onto a $6 \%$ SDS-PAGE and separated by electrophoresis, followed by transfer onto polyvinylidene difluoride membranes (EMD Millipore,
Billerica, MA, USA). Following blocking with 5\% skimmed milk in Tris-buffered saline containing $0.1 \%$ Tween-20 at room temperature for $1 \mathrm{~h}$, the membranes were probed at $4^{\circ} \mathrm{C}$ overnight with the following primary antibodies: Rabbit anti-FASN (cat. no. A6273; dilution, 1:500; ABclonal Biotech Co., Ltd., Woburn, MA, USA), anti-DNA-dependent protein kinase catalytic subunit (DNA-PKcs; cat. no. 4602; dilution, 1:500; Cell Signaling Technology, Inc.) and mouse anti- $\beta$-actin (cat. no. TA811000; dilution, 1:10,000; OriGene Technologies, Inc., Beijing, China). The membranes were washed using tris buffered saline with $0.1 \%$ Tween 20 three times prior to incubation with horseradish peroxidase-conjugated goat anti-rabbit IgG (cat. no. A0208; Beyotime Institute of Biotechnology, Haimen, China; 1:2,000 dilution) for $1 \mathrm{~h}$ at room temperature. The proteins were visualized using a SuperSignal enhanced chemiluminescence kit (Thermo Fisher Scientific, Inc.).

Statistical analysis. Each experiment was repeated at least three times, and statistical analyses were performed using SPSS (version 22.0; IBM Corp., Armonk, NY, USA). The results are expressed as the mean \pm standard deviation, and statistically significant differences were analyzed using one-way analysis of variance, followed by the Tukey's post hoc tests. $\mathrm{P}<0.05$ was considered to indicate a statistically significant difference.

\section{Results}

Lentivirus-mediated shRNA interference inhibits FASN expression in A549 cells. To assess the role of FASN in A549 cells, FASN expression was knocked down using a lentivirus-mediated shRNA approach, and the FASN mRNA and protein expression levels in transfected cells were evaluated using RT-qPCR and western blot analysis. The results of RT-qPCR analysis revealed that FASN mRNA levels were suppressed by $\sim 92$ and $88 \%$ in the cells that were transfected with shFASN\#3072 and shFASN\#7377, respectively. FASN mRNA was significantly decreased in shFASN\#3072 and shFASN\#7377-transfected cells compared with the negative control (NC) group $(\mathrm{P}<0.01$; Fig. 1A). The RT-qPCR results 
were consistent with the results of the western blot analysis (Fig. 1B), which revealed that the expression of FASN protein in cells transfected with shFASN\#3072 or shFASN\#7377 was markedly diminished compared with the NC group. These data suggested that the lentivirus-mediated shRNA system was able to successfully inhibit the expression of FASN in A549 cells.

Knockdown of FASN inhibits the proliferation of A549 cells. The effect of FASN knockdown on cell proliferation was investigated using a CCK- 8 assay. After 1, 2 and 3 days, the absorbance values of the cells that were transfected with FASN-shRNA\#3072 and FASN-shRNA\#7377 revealed that proliferation was significantly inhibited in these groups compared with the NC group. The proliferation rates of shFASN\#3072- and shFASN\#7377-transfected cells at 24, 48 and $72 \mathrm{~h}$ were decreased by 16, 29 and 27\% (shFASN\#3072; $\mathrm{P}<0.01$ ), and 18, 17 and 17\% (shFASN\#7377; $\mathrm{P}<0.05)$, respectively (Fig. 2).

Radiosensitivity is increased in A549 cells transfected with FASN shRNA. To determine the effect of FASN expression on the radiosensitivity of A549 cells, colony formation assays were performed following exposure of the cells to varying doses of irradiation. As demonstrated in Fig. 3, the number of colonies formed by cells transfected with shFASN\#3072 and shFASN\#7377 was significantly reduced compared with the NC group. In addition, radiation led to a dose-dependent decrease in the survival of $\mathrm{A} 549$ cells $(\mathrm{P}<0.05$ for all radiation doses). The results indicated that shRNA-FASN cells exhibited reduced radioresistance compared with the cells in the $\mathrm{NC}$ group.

Silencing FASN increases the percentage of cells in the G2/M phase following irradiation. Flow cytometric analysis was conducted to evaluate changes in cell cycle progression following irradiation. As indicated in Fig. 4, the groups transfected with shFASN\#3072 and shFASN\#7377 exhibited a higher proportion of cells in the G2/M phase compared with the NC group (shFASN\#3072 and shFASN\#7377 vs. NC group, $9.93 \pm 1.56 \%$ and $8.13 \pm 1.62 \%$ vs. $3.82 \pm 0.17 \%$; $\mathrm{P}<0.05$; Fig. 4) Following exposure to 6 Gy irradiation, the proportion of cells in the $\mathrm{G} 2 / \mathrm{M}$ phase was significantly increased in shFASN\#3072 and shFASN\#7377-transfceted cells compared with negative control (shFASN\#3072 and shFASN\#7377 vs. NC group, $18.79 \pm 0.97 \%$ and $15.16 \pm 1.53 \%$ vs. $5.50 \pm 0.49 \%$; $P<0.01$; Fig. 4). These data suggested that transfection of FASN-shRNA combined with ionizing radiation increased cell cycle arrest at the $\mathrm{G} 2 / \mathrm{M}$ phase compared with the NC group.

Downregulation of FASN increases radiation-induced apoptosis of A549 cells. The rate of apoptosis following ionizing radiation treatment was evaluated using Annexin V/PI staining. As indicated in Fig. 5, flow cytometry revealed that the proportion of apoptotic cells in the cells transfected with shFASN\#3072 or shFASN\#7377 was higher compared with the $\mathrm{NC}$ group. Following treatment with $6 \mathrm{~Gy}$ radiation, the rate of apoptosis in cells transfected with shFASN\#3072 or shFASN\#7377 was significantly increased compared with the NC group $(\mathrm{P}<0.01)$. The percentages of apoptotic cells (upper and lower right quadrants) for shFASN\#3072- and

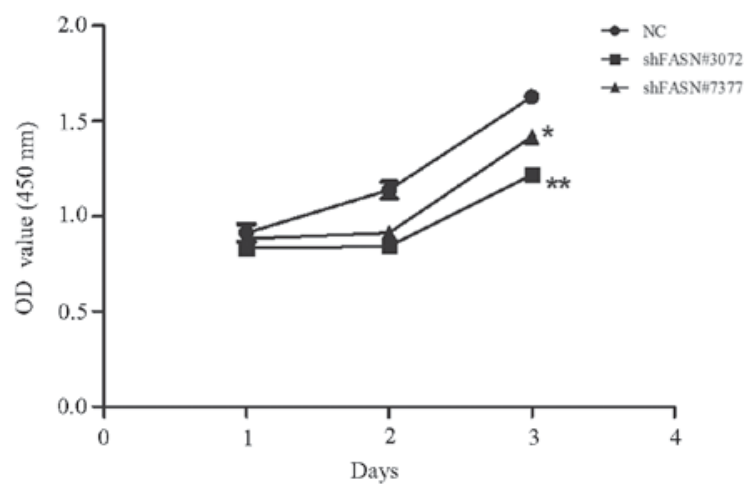

Figure 2. Knockdown of FASN by shRNA inhibits proliferation of A549 cells. At the indicated time points, the cells that were transfected with vectors containing shFASN\#3072, shFASN\#7377 or NC were analyzed using cell counting kit- 8 assay in triplicate samples. ${ }^{*} \mathrm{P}<0.05$ and ${ }^{* *} \mathrm{P}<0.01$ vs. NC. FASN, fatty acid synthase; shRNA, short hairpin RNA; OD, optical density; $\mathrm{NC}$, negative control.

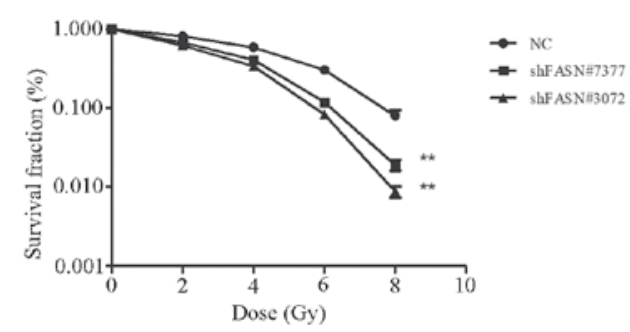

Figure 3. Survival curve of A549 cells following exposure to various doses of ionizing radiation, as evaluated by a colony formation assay. Knockdown of FASN by shRNA was able to significantly increase the radiosensitivity in A549 cells that were transfected with shFASN\#3072 and shFASN\#7377. ${ }^{* *} \mathrm{P}<0.01$ vs. NC. shRNA, short hairpin RNA; NC, negative control.

shFASN\#7377-transfected and NC cells without radiation treatment were $9.8 \pm 1.47,8.4 \pm 1.21$ and $3.93 \pm 0.47 \%$, respectively and the values following treatment with $6 \mathrm{~Gy}$ radiation for shFASN\#3072- and shFASN\#7377-transfected and NC cells were $15.38 \pm 1.00,27.79 \pm 2.25$ and $19.85 \pm 1.34 \%$, respectively. These data indicated that NSCLC cells transfected with FASN-shRNA that were subjected to ionizing radiation had an increased rate of apoptosis compared with the NC group.

Downregulation of FASN inhibits DNA damage repair following irradiation. To analyze whether the inhibition of FASN may affect radiosensitivity, the expression levels of DNA-PKcs protein, a key protein associated with the repair of DNA DSBs, following exposure to 6 Gy irradiation were analyzed. As indicated in Fig. 6, the downregulation of FASN expression suppressed the levels of DNA-PKcs and inhibited the increase in the levels of these proteins following irradiation. These results indicated that FASN-mediated radioresistance may be associated with the expression of the DNA DSB repair protein DNA-PKcs.

\section{Discussion}

Numerous previous studies have demonstrated that FASN, a multifunctional enzyme, has important effects on the 
A

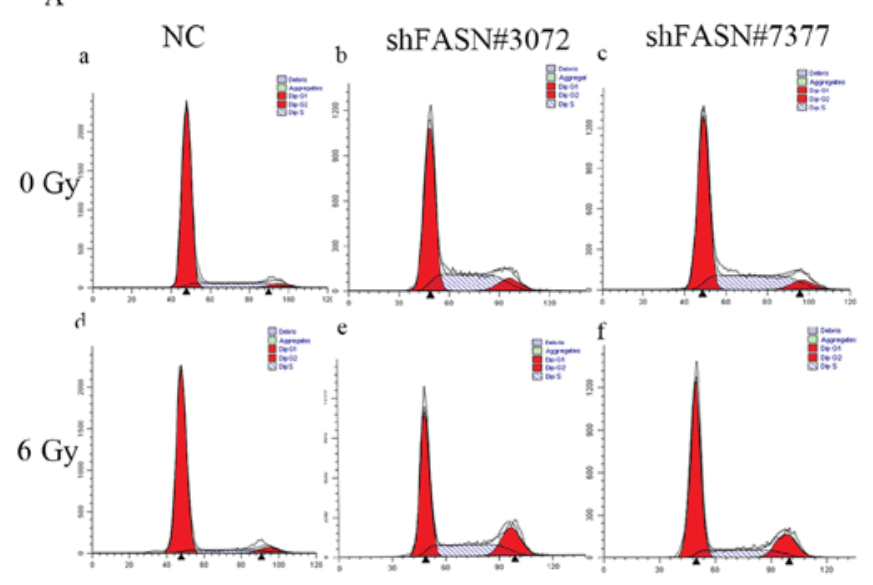

B

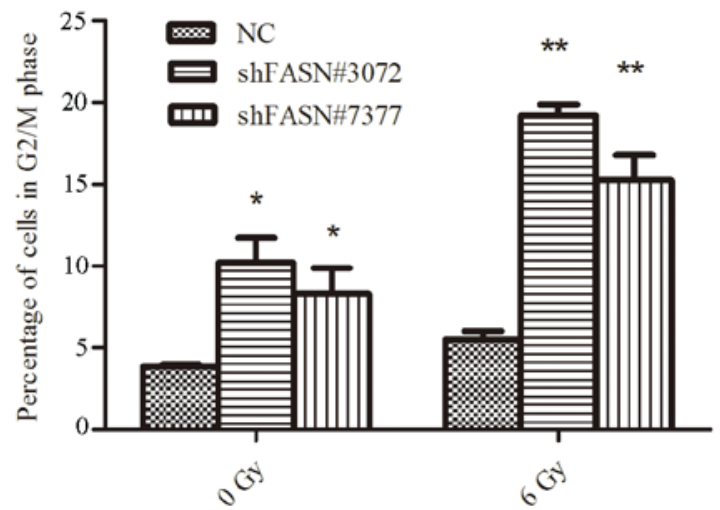

Figure 4. Effect of shFASN and ionizing radiation on the proportion of cells in the G2/M phase. (A) The effects of FASN knockdown and 6 Gy irradiation on cell cycle distribution of A549 cells, as determined by flow cytometry using propidium iodide staining. (B) The percentage of A549 cells in the G2/M phase. ${ }^{*} \mathrm{P}<0.05$ and $^{* *} \mathrm{P}<0.01$ vs. NC. FASN, fatty acid synthase; shRNA, short hairpin RNA; NC, negative control.
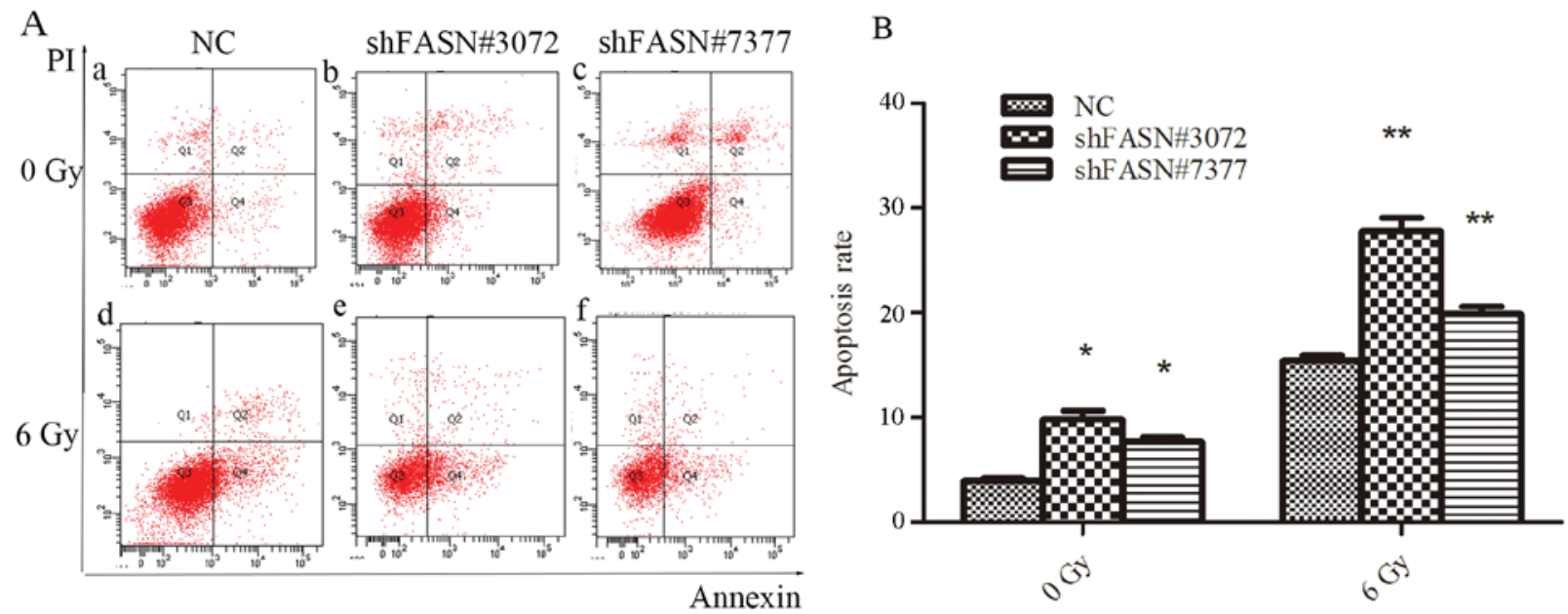

Figure 5. Transfection with shFASN and ionizing irradiation promotes radiation-induced apoptosis in A549 cells. (A) The effects of FASN knockdown on apoptosis following 6 Gy irradiation in A549 cells. The apoptosis rate was determined by flow cytometry using Annexin V/PI staining. (B) The apoptotic rate of shFASN and NC cells with and without radiation treatment. Apoptosis was significantly increased in cells transfected with shFASN. ${ }^{*} \mathrm{P}<0.05$ and ${ }^{* *} \mathrm{P}<0.01$ vs. NC. FASN, fatty acid synthase; shRNA, short hairpin RNA; PI, propidium iodide; NC, negative control.

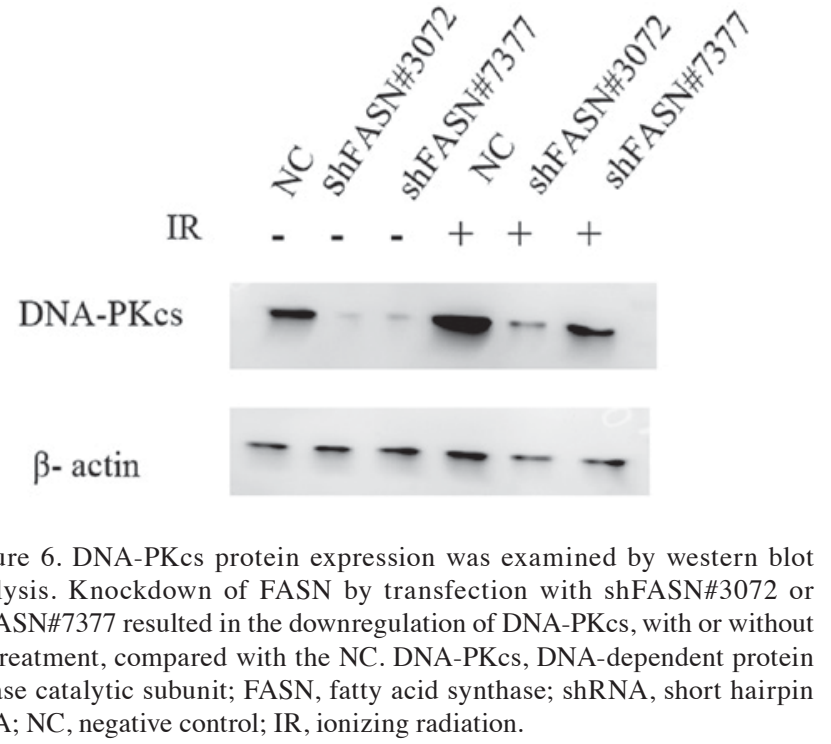

occurrence, progression, invasion and metastasis of tumors (15). In mammalian metabolism, fatty acids are either exogenously derived from the diet or endogenously derived via de novo lipogenesis (19). Under physiological conditions, due to a balanced diet, FASN is thought to provide the fatty acids for growth, development and survival (20). Therefore, the latter is the primary source of fatty acids (21). FASN is a $250-270-\mathrm{kDa}$ cytosolic protein, and its main physiological function is to catalyze the production of palmitate, a 16-carbon saturated fatty acid, from acetyl CoA and malonyl CoA (22). However, little is known about its function in radiosensitivity of NSCLC. The aim of the present study was to investigate the effect of FASN knockdown by constructing a lentiviral vector containing shRNA that targets FASN (pSIH-H1-Puro-shRNA-FASN). The results demonstrated that FASN is associated with the regulation of cell proliferation, cell cycle and apoptosis. In addition, FASN expression was revealed to have a significant effect on radiosensitivity, where the downregulation of FASN 
increased the sensitivity of A549 cells to radiation. These results are in agreement with those of Yang et al (17), who recently reported that FASN expression was correlated with radiation resistance and poor clinical outcome in patients with pancreatic cancer.

FASN may be an optimal diagnostic marker and a potential therapeutic target due to its distinctive tissue distribution and special enzymatic activity $(23,24)$. FASN is often highly expressed in human cancer, whereas it is usually undetectable or exhibits low expression in the majority of normal human tissues (13-17). This differential tissue distribution makes FASN an attractive target for the development of novel anticancer techniques (13-16). Zhou et al evaluated 80 samples of esophageal cancer, revealing that FASN is primarily localized in the cytoplasm of esophageal cancer, but exhibited little or no expression in normal esophageal tissues (16). Similarly, Kao et al (15) investigated FASN expression in nasopharyngeal carcinoma using immunohistochemistry and revealed that FASN was positively expressed in the tumoral cytoplasm, but exhibited low or no expression in non-tumor epithelium.

Previous studies have demonstrated that the upregulation of FASN increases tumor cell proliferation (25), decreases the rate of apoptosis (17), and increases the resistance to chemotherapy and radiation of tumor cells in various human neoplasms (26). In line with this, the present study revealed that downregulation of FASN caused a significant reduction in the proliferation of A549 cells, suggesting that FASN may be involved in NSCLC oncogenesis. Furthermore, silencing of FASN led to cell cycle arrest in the G2/M phase, suggesting that FASN promotes proliferation through modulation of cell cycle. Furthermore, silencing of FASN was accompanied by an increase in apoptosis. Similarly, a previous study by Jiang et al (14) demonstrated that downregulation of FASN led to decreased proliferation and increased apoptosis in bladder cancer cells. Notably, the combination of shFASN and ionizing radiation significantly inhibited proliferation, promoted apoptosis and increased cell cycle arrest in the $\mathrm{G} 2 / \mathrm{M}$ phase compared with shFASN or ionizing radiation alone.

To address the fact that a decrease in apoptosis may lead to long-term resistance to radiotherapy, a colony formation assay was conducted in the present study, revealing that transfection with shFASN led to the formation of considerably fewer colonies compared with the control group. This indicates that the knockdown of FASN is able to markedly enhance cell susceptibility to irradiation. At the same time, this demonstrated that a combination of shFASN and ionizing radiation reduced clonogenic survival compared with each treatment alone.

The present study demonstrated that the knockdown of FASN expression increased the radiosensitivity of A549 cells. Additionally, previous studies have indicated that FASN overexpression contributes to the resistance to several chemotherapeutics $(17,26)$. This finding prompted the investigation into whether or not FASN regulates DNA damage responses.

DNA is a major target that is damaged by ionizing irradiation, generating a series of genomic DNA lesions, of which DSBs are the most important (27). There are two major mechanisms for the repair of DSBs induced by ionizing radiation: Homologous recombination and non-homologous end joining (NHEJ) (28). NHEJ is regarded as the primary mechanism for the repair of radiation-induced DSBs throughout the cell cycle (29). In healthy cells, this repair function is crucial for cell survival, while in cancer cells this may lead to resistance to radiation (30). The DNA-PK proteins, which are involved in NHEJ, contain DNA-PKcs and ku70/ku80 (31). DNA-PKcs has been verified to initiate DNA repair directly by combining with DNA ends in the absence of ku70/ku80 (32). As discussed earlier, the present study assessed the association between FASN and DNA-PKcs in order to investigate the mechanism by which FASN affects the radiosensitivity of NSCLC cells. The results from western blotting revealed that the inhibition of FASN downregulated the protein levels of DNA-PKcs and markedly inhibited the increase of the expression of this protein following irradiation. This finding indicates that the suppression of FASN expression may decrease the ability of NSCLC cells to repair DSBs induced by radiation and that this effect is partly mediated by the DNA-PKcs pathway. Further experiments to analyze the possible molecular mechanisms of DNA DSB repair proteins in the regulation of FASN-mediated radiosensitivity are currently being conducted in the laboratory of the present authors.

In summary, the present study demonstrated that the suppression of FASN combined with ionizing radiation enhanced the radiosensitivity of NSCLC cells by inhibiting proliferation, promoting cell cycle arrest, triggering apoptosis and decreasing the DNA damage repair capability of A549 cells. Therefore, combined with the observations reported in previous studies, this suggests that FASN may be a potential target for therapeutic interventions designed to increase the radiosensitivity of NSCLC.

\section{References}

1. Takanen S, Bangrazi C, Graziano V, Parisi A, Resuli B, Simione L, Caiazzo R, Raffetto N and Tombolini V: Number of mediastinal lymph nodes as a prognostic factor in PN2 non small cell lung cancer: A single centre experience and review of the literature. Asian Pac J Cancer Prev 15: 7559-7562, 2014.

2. Tarone RE: On the International Agency for Research on Cancer classification of glyphosate as a probable human carcinogen. Eur J Cancer Prev: Nov 8, 2017 (Epub ahead of print).

3. Parsons A, Daley A, Begh R and Aveyard P: Influence of smoking cessation after diagnosis of early stage lung cancer on prognosis: Systematic review of observational studies with meta-analysis. BMJ 340: b5569, 2010.

4. Jemal A, Bray F, Center MM, Ferlay J, Ward E and Forman D: Global cancer statistics. CA Cancer Clin 61: 69-90, 2011.

5. Fernandes AT, Mitra N, Xanthopoulos E, Evans T, Stevenson J, Langer C, Kucharczuk JC, Lin L and Rengan R: The impact of extent and location of mediastinal lymph node involvement on survival in Stage III non-small cell lung cancer patients treated with definitive radiotherapy. Int J Radiat Oncol Biol Phys 83: 340-347, 2012

6. Shikazono N, Noguchi M, Fujii K, Urushibara A and Yokoya A: The yield, processing, and biological consequences of clustered DNA damage induced by ionizing radiation. J Radiat Res 50: 27-36, 2009.

7. Greenstein JP: Biochemistry of cancer. New York: Academic Press, 1954

8. Nakashima RA, Paggi MG and Pedersen PL: Contributions of glycolysis and oxidative phosphorylation to adenosine 5'-triphosphate production in AS-30D hepatoma cells. Cancer Res 44: 5702-5706, 1984

9. Medes G, Thomas A and Weinhouse S: Metabolism of neoplastic tissue. IV. A study of lipid synthesis in neoplastic tissue slices in vitro. Cancer Res 13: 27-29, 1953.

10. Chung YW, Han DS, Park YK, Son BK, Paik CH, Lee HL, Jeon YC and Sohn JH: Association of obesity, serum glucose and lipids with the risk of advanced colorectal adenoma and cancer: a case-control study in Korea. Dig Liver Dis 38: 668-672, 2006. 
11. Williams RR, Shorlie PD and Feinleib M: Cancer incidence by levels of cholesterol. JAMA 245: 247-25234, 1981.

12. Liu H, Liu JY, Wu X and Zhang JT: Biochemistry, molecular biology, and pharmacology of fatty acid synthase, an emerging therapeutic target and diagnosis/prognosis marker. Int J Biochem Mol Biol 1: 69-89, 2010.

13. Khan A, Aljarbou AN, Aldebasi YH, Faisal SM and Khan MA: Resveratro suppresses the proliferation of breast cancer cells by inhibiting fatty acid synthase signaling pathway. Cancer Epidemiol 38: 765-772, 2014.

14. Jiang B, Li EH, Lu YY, Jiang Q, Cui D, Jing YF and Xia SJ: Inhibition of fatty acid synthase supresses P-akt and induces apoptosis in bladder cancer. Urology 80: 484.e9-e15.

15. Kao YC, Lee SW, Lin LC, Chen LT, Hsing CH, Hsu HP, Huang HY, Shiue YL, Chen TJ and Li CF: Fatty acid synthase overexpression confers an independent prognosticator and associates with radiation resistance in nasopharyngeal carcinoma. Tumor Biol 34: 759-768, 2013.

16. Zhou Y, Niu C, Li Y, Gao B, Zheng J, Guo X and Ma W: Fatty acid synthase expression and esophageal cancer. Mol Biol Rep 39: 9733-9739, 2012.

17. Yang Y, Liu H, Li Z, Zhao Z, Yip-Schneider M, Fan Q, Schmidt CM, Chiorean EG, Xie J, Cheng L, et al: Role of fatty acid synthase in gemcitabin and radiation resistance of pancreatic cancer. Int J Biochem Mol Biol 2: 89-98, 2011

18. Livak KJ and Schmittgen TD: Analysis of relative gene expression data using real-time quantitative PCR and the 2(-Delta Delta C(T)) method. Methods 25: 402-408, 2001

19. Menendez JA and Lupu R: Fatty acid synthase and the lipogenic phenotype in cancer. Nat Rev Cancer 7: 763-77, 2007.

20. Shaw RJ: Glucose metabolism and cancer. Curr Opin Cell Biol 18: 598-608, 2006.

21. Kuhajda FP: Fatty-acid Synthase and human cancer: New perspectives on its role in tumor biology. Nutrition 16: 202-208, 2000.

22. Smith S, Witkowski A and Joshi AK: Structural and functional organization of the animal fatty acid synthase. Prog Lipid Res 42: 289-317, 2003.

23. Pizer ES, Thupari J, Han WF, Pinn ML, Chrest FJ, Frehywot GL, Townsend CA and Kuhajda FP: Malonyl-coenzyme-A is a potential mediator of cytotoxicity induced by fatty-acid synthase inhibition in human breast cancer cells and xenografts. Cancer Res 60: 213-218, 2000.
24. De Schrijver E, Brusselmans K, Heyns W, Verhoeven G and Swinnen JV: RNA interference-mediated silencing of the fatty acid synthase gene attenuates growth and induces morphological changes and apoptosis of LNCaP prostate cancer cells. Cancer Res 63: 3799-3804, 2003.

25. Yoshii Y, Furukawa T, Oyama N, Hasegawa Y, Kiyono Y, Nishii R, Waki A, Tsuji AB, Sogawa C, Wakizaka H, et al: Fatty acid synthase is a key target in multiple essential tumor functions of prostate cancer: Uptake of radiolabeled acetate as a predictor of the targeted therapy outcome. PLoS One 8: e64570, 2013.

26. Liu H, Wu X, Dong Z, Luo Z, Zhao Z, Xu Y and Zhang JT: Fatty acid synthase causes drug resistance by inhibiting TNF- $\alpha$ and ceramide production. J Lipid Res 54: 776-785, 2013.

27. Yanold J: Molecular aspects of cellular responses to radiotherapy. Radiothery Oncol 44: 1-7, 1997.

28. Thompson LH: Evidence that mammalian cells possess homologous recombinational repair pathways. Mutat Res 363: 77-88, 1996.

29. Bertolini LR, Bertolini M, Anderson GB, Maga EA, Madden KR and Murray JD: Transient depletion of Ku70 and Xrcc 4 by RNAi as a means to manipulate the non-homologous end-joining pathway. J Biotechnol 128: 246-257, 2007.

30. Hamer G, Roepers Gajadien HL, Van Duyn-Goedhart A Gademan IS, Kal HB, van Buul PP, Ashley T and de Rooij DG: Function of DNA-protein kinase catalytic subunit during the early meiotic prophase without Ku70 and Ku86. Biol Reprod 68: 717-721, 2003.

31. Jeggo PA: Identification of genes involved in repair of DNA doublestrand breaks in mammary cells. Radiat Res 150: 580-591, 1998.

32. Yaneva M, Kowalewski $\mathrm{T}$ and Lieber MR: Interaction of DNA-dependent protein kinase with DNA and with $\mathrm{Ku}$ : Biochemical and atomic force microscopy. EMBO J 16: 5098-5112, 1997. 